\title{
Access Control Model Based on RDB Security Policy for OWL Ontology*
}

\author{
Dongwon Jeong ${ }^{1}$, Yixin Jing ${ }^{2}$, and Doo-Kwon Baik ${ }^{2}$ \\ ${ }^{1}$ Dept. of Informatics \& Statistics, Kunsan National University, \\ San 68, Miryoung-dong, Gunsan, Jeollabuk-do, 573-701 Korea \\ djeong@kunsan.ac.kr \\ ${ }^{2}$ Dept. of Computer Science \& Engineering, Korea University, \\ Anam-dong, Sungbuk-gu, Seoul, 136-701 Korea \\ \{jing, baik\} @software.korea.ac.kr
}

\begin{abstract}
Most of information has been storing and managing in relational databases and there are many researches to store OWL ontologies. In this situation, a study on efficient access control model using relational security model is required. The paper proposes a novel access control model for OWL ontologies in relational database systems. The access control is realized through evaluating queries against an OWL data view. On one hand, the OWL data view prevents the sensitive information revealed to unauthorized users. On the other hand it considers the inference ability of users. An empirical study verifies the effectiveness of our approach.
\end{abstract}

\section{Introduction}

Web Ontology Language (OWL) is a knowledge description language and is recognized as one of the most important technologies to realize Semantic Web [1]. Compared to XML, OWL supports more enhanced impression power and enables machines to infer new knowledge. There is much effort on how to build and store OWL knowledge bases (Ontologies, Documents). However, few of research pay attention to the OWL document security issue. This may result in knowledge leakage and also makes high-quality services through secure knowledge access so hard.

This paper contributes a novel OWL security (Access Control) model using relational database security model. It means that we assume OWL knowledge bases are stored in a relational database. There might be a question why we propose an access control model for OWL knowledge bases in relational databases. The reasons are: (1) most of information has been managing by relational database systems; (2) relational database systems provide a secure and stable access control model.

Therefore, we propose a security model based on relational databases considering this reality. In other words, for enforcement of access control to the OWL knowledge bases, the persistent storage should be taken into consideration. The prevalent

* This work was supported by the Korea Research Foundation Grant funded by the Korean Government(MOEHRD) (KRF-2006-311-D00776). 
approaches can be classified into two types: (1) File-based storage model; (2) Persistent storage model (e.g., RDB-based storage). The file-based storage models, such as Jena [2] and Protege [3], are based on the graph model (Triple structure). Jena provides a persistent storing method to manage OWL knowledge in relational databases. However, it still suffers from the inefficiency.

To solve this problem, we first define a new database layout to efficiently store OWL knowledge. In this paper, our proposal on OWL ontology data access control is outlined as follows: (1) First, we design an efficient relational data model to store OWL data persistently. Our model gains predominant over Jena2; (2) Second, we define a new OWL security model using relational access control model; (3) Finally, we show the evaluation results through experiments.

\section{Persistent OWL Storage Model in Relational Databases}

\subsection{OWL-DL Ontology Model}

We briefly review OWL-DL document. Without loss of generality, we represent an OWL ontology by Definition 1.

Definition 1. OWL-DL ontology is composed of (N, P, literal),

Predicate $\mathrm{P}: \mathrm{N} \rightarrow \mathrm{N}$ I $\mathrm{N} \rightarrow$ literal

type_n: $\mathrm{N} \rightarrow$ \{NamedClass, Instance, Non-NamedClass $\}$

type_p: $\mathrm{P} \rightarrow\{$ Transitive, Non-Transitive $\}$

type_p $\left(\mathrm{p}_{\mathrm{c}}:=\right.$ subClassOf $)=$ Transitive

We consider the ontology as a graph. $\mathrm{N}$ and $\mathrm{P}$ represent nodes and edges respectively. $\mathrm{P}$ is a one to one mapping from $\mathrm{N}$ to $\mathrm{N}$, or from $\mathrm{N}$ to literal. In the context of $\mathrm{OWL}$ $\mathrm{DL}, \mathrm{N}$ is a set of subclasses and instances of RDFNode, while $\mathrm{P}$ is a set of subclasses and instances of RDFProperty. Function type_n and type_p are many-to-one mapping and return the type of element of $\mathrm{N}$ and $\mathrm{P}$ respectively. As for any $\mathrm{n}_{\mathrm{i}}$ in $\mathrm{N}, \mathrm{n}_{\mathrm{i}}$ type is either NamedClass, Instance, or Non-NamedClass. NamedClass are those classes which are given explicit URIs in the OWL document. Non-NamedClass includes such as ClassExpression, Restriction and AnonymousClass defined in the OWL syntax. As for any $\mathrm{p}_{\mathrm{i}}$ in $\mathrm{P}$, the pi type is either Transitive or Non-Transitive. If a $\mathrm{p}_{\mathrm{i}}$ is transitive, $\mathrm{p}_{\mathrm{i}}\left(\mathrm{n}_{1}, \mathrm{n}_{2}\right) \wedge \mathrm{p}_{\mathrm{i}}\left(\mathrm{n}_{2}, \mathrm{n} 3\right) \rightarrow \mathrm{p}_{\mathrm{i}}\left(\mathrm{n}_{1}, \mathrm{n}_{3}\right)$. Particularly if a $\mathrm{p}_{\mathrm{c}}$ is rdfs:subClassOf, its type is of Transitive.

In addition, we define two functions to facilitate the query evaluation algorithm. $\operatorname{asDomain}\left(\mathrm{n}_{\mathrm{i}}\right)$ returns a set of properties which domain is $\mathrm{n}_{\mathrm{i}}$; asRange $\left(\mathrm{n}_{\mathrm{i}}\right)$ returns a set of properties which range is n. Take the OWL file in Fig. 1 as an example, which is shown as a graph. The RedWine is the subclass of Wine and a restriction, which constrains that property hasColor refers to Red. type_p(subClass) is Transitive, other properties are Non-Transitive.

\subsection{Permanent Storage Model}

The goal of this paper is to define a novel OWL access control model based on the relational database security model. It means that we an efficient database layout to 
store OWL knowledge base and to provide an efficient way to facilitate access control. First, the named Classes in an OWL document are identified. According to each named Class's definition, its nesting Restriction is saved in different tables. The layout also saves the instances of each named Class. The Property table created connections between the Classes by specifying property domain and range. Through testing with lots of existing OWL ontologies, the persistent relational model is proved capable of saving any general OWL data.

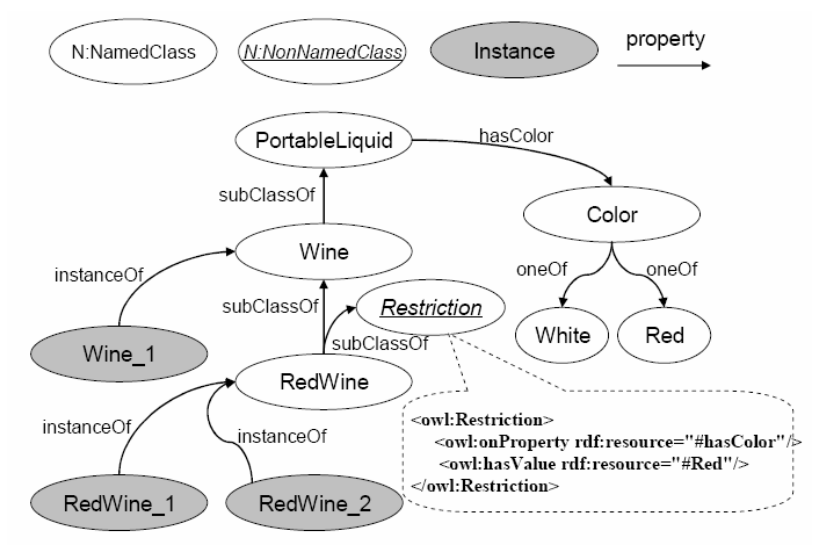

Fig. 1. A simple OWL ontology

Fig. 2 shows a metamodel of the relational model for storing persistently. An OWL ontology basically consists of a triple (Subject, Predicate, Object). In this paper, both of Subject and Object are described as Concept or Instance. In the OWL specification, they are defined as Class and Individual.

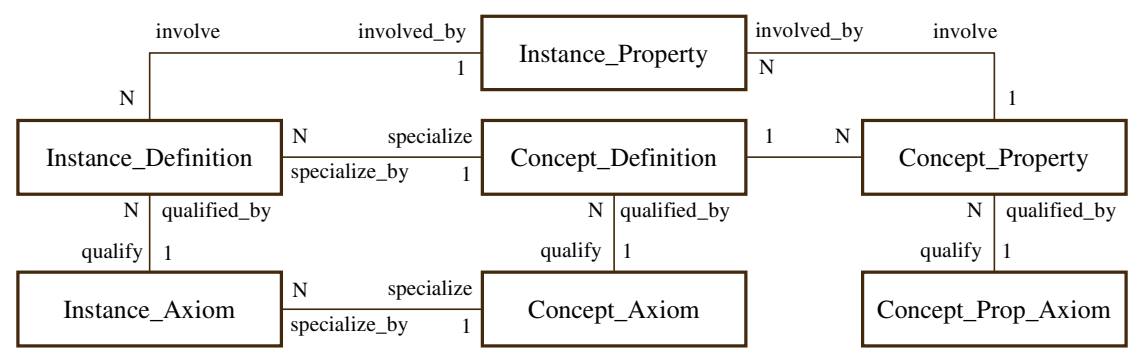

Fig. 2. Metamodel for efficiently storing OWL ontology

\section{OWL Security Model}

\subsection{Definition of Access Control Model}

In this section, we define the OWL data access control model. This concept model provides a general definition. 
Definition 2. OWL Access Control Model: $M=(P, V, R)$, where P: OWL data in a persistent storage (RDB);

V: OWL data view set; and R: Role set.

$\mathrm{V}$ is used to constrain the OWL data that a role can obtain. For each role, there is only one view connected with it. User can access to the $\mathrm{P}$ through one of $\mathrm{V}$ by playing a role. In addition, we define the function $\varphi: V \rightarrow R$ to return a data view assigned to a role. $\varphi$ is a many to one function. According the definition 2, a framework for the propose security model is illustrated in Fig. 3.

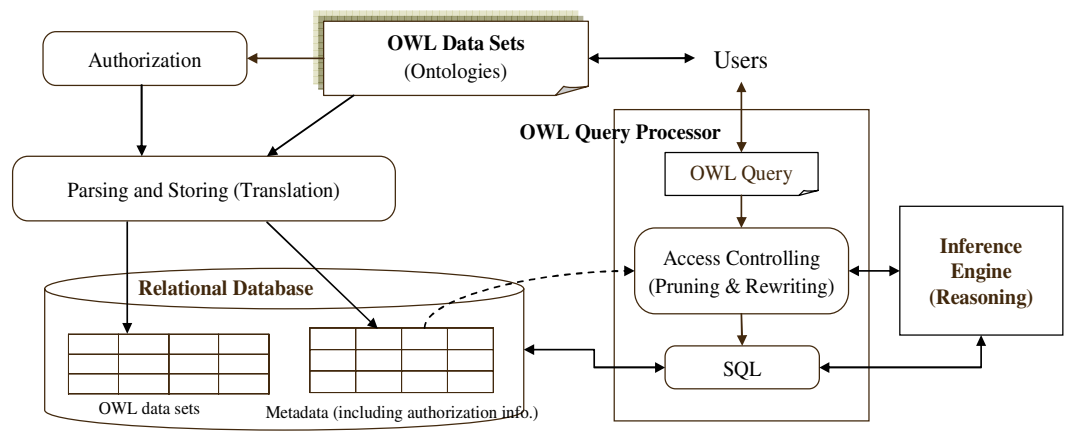

Fig. 3. A framework for the proposed security model

\subsection{Views for OWL Ontology}

Although an OWL ontology is successfully ported into a relational database system, the attempt to adopt the relational database security solution encounters challenges. Creating view on the tables can only obtain an access control in a very limited level. Only the limit to the column access is not enough. Access control on OWL data needs to evaluate the query to individual record such as Wine or RedWine, which requires the access control on the record level. The security view on OWL data consists of two parts for securing $\mathrm{N}$ and $\mathrm{P}$ respectively.

Definition 3. OWL Data View: $V=\left(V_{N}, V_{P}\right)$,

where $V_{\mathrm{N}}:\left\{\mathrm{n}_{\mathrm{i}}\right\} \rightarrow\left\{\right.$ false, (true, $\wedge$ transitiveEnd $\left.\left.\left.\left(\mathrm{p}_{\mathrm{i}}\right)=\left[\mathrm{n}_{\mathrm{s}}, \mathrm{n}_{\mathrm{e}}\right]\right\}\right)\right\}$ $\mathrm{V}_{\mathrm{P}}:\left\{\mathrm{p}_{\mathrm{i}}\right\} \rightarrow$ false, true $\}$

$\mathrm{V}_{\mathrm{N}}$ maps each node $\mathrm{n}_{\mathrm{i}}$ in ontology graph to a security specification for a given user. The specification denotes a $n_{i}$ inaccessible (false), or accessible (true) along with a constraint to transitive end. Transitive end constrains the inference ability from the node. From the perspective of a graph, the inference ability can be considered as the ability of how far the current node can access tracking along with a transitive property. The TransitiveEnd defines the end of this inference path. $\left[\mathrm{n}_{\mathrm{s}}, \mathrm{n}_{\mathrm{e}}\right]$ denotes end 
nodes where the inference path comes to an end in two directions. The end node can be Self, another node or Full. Self means the inference stop at the node itself. Full doesn't restrict the inference ability. VP denotes the access to a given property pi. An example $\mathrm{V}_{\mathrm{N}}($ RedWine $)=($ true, trasitiveEnd(subClassOf $)=$ [self, Wine]) presents a view constraining that a user can visit RedWine and can only visit its super classes till Wine.

\section{Experiment and Evaluation}

A query is a request for accessing a given content in ontology. A query pattern is an abstract template and a query (concrete query) request the desirable OWL information by adopting this template. The query pattern and query can be defined as Definition 4 and Definition 5.

Definition 4. Query Pattern $\mathrm{P}_{\mathrm{Q}}=\left(\wedge_{1<i<=|\mathrm{N}|}\left(\mathrm{p}_{\mathrm{x}}\left(? \mathrm{x}, \mathrm{n}_{\mathrm{i}}\right) \mid \mathrm{p}_{\mathrm{y}}\left(\mathrm{n}_{\mathrm{j}}, ? \mathrm{x}\right)\right)\right)$, where $\mathrm{n}_{\mathrm{i}} \in$ Nlliteral, $\mathrm{n}_{\mathrm{j}} \in \mathrm{N}, \mathrm{p}_{\mathrm{x}}, \mathrm{py} \in \mathrm{P}$.

Each element of the conjunction is called a constraint. $n_{i}, n_{j}, p_{x}$, and $p_{y}$ are named condition variables. ?x is output variable.

Definition 5. Query $q=\left[\mathrm{P}_{\mathrm{Q}}\right]$ is a value assignment to condition variables in query pattern $\mathrm{P}_{\mathrm{Q}}$.

For example, a query get the instances of Car which are made in USA can be expressed as $\mathrm{q}=($ instanceOf(?x, Car) $\wedge$ make(USA, ?x )), constraints are instanceOf(?x, Car) and make(USA, ?x ). When a user issue a query, the query evaluation will first get the OWL view according to the user's role through $\varphi$, then consult the view. If the role is authorized to get access to all condition variables and output variable, the satisfying result is returned. Moreover, query evaluation is responsible to generate SQL query to the underlying database.

This paper assumes that the translation from OWL data query in query pattern to database compliant SQL is possible. The query evaluation algorithm Query_Eva (V, q) is shown in Fig. 4.

As an example, consider the query $q=$ instanceOf(RedWine_1, ?x) along with the VN(RedWine). Since VN(RedWine) defines the super classes of RedWine is visible until Wine, and the other content on ontology is fully accessible, the algorithm returns a graph consists of RedWine and Wine, without PortableLiquid. If the same query is processed with normal reasoning engine without OWL data view, the result would include PortableLiquid. Our proposal offers a controllable reasoning approach. Another example query is instanceOf(?x, PortableLiquid). If $\mathrm{VN}($ PortableLiquid $)=($ true, trasitiveEnd $($ subClassOf $)=[$ Wine, self $])$, the returned graph includes Wine_1, without the instances of RedWine. The evaluated result is illustrated in Fig. 5. 


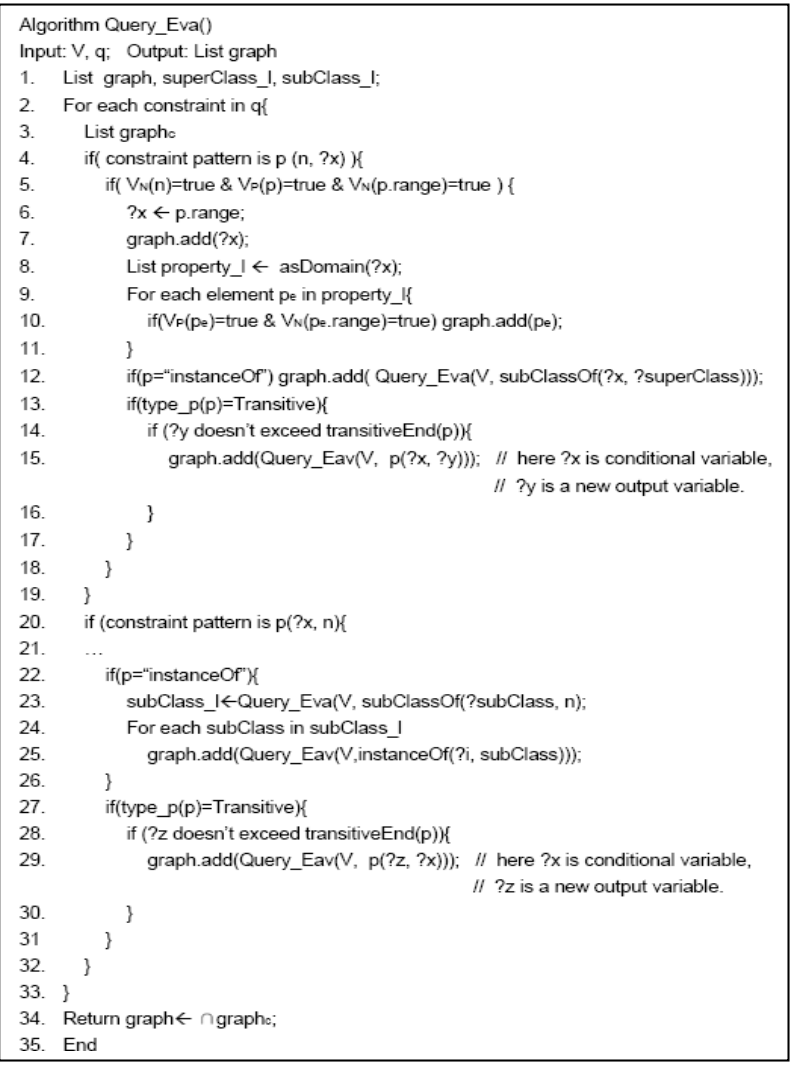

Fig. 4. Query evaluation algorithm

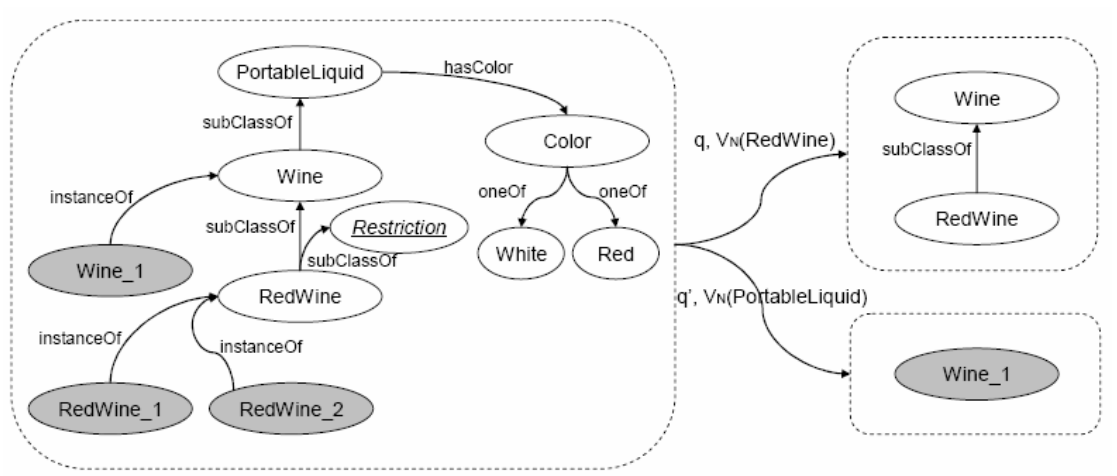

Fig. 5. Query evaluation result

In the experiment, we put focus on the effect that the number of query constraints and transitive property take on the efficiency. To exactly investigate the relationship 
between the numbers of query constraints with time-consuming, we tested a query, which constraints number is from 100 to 1000, and the constraints' properties are all non-transitive.

Fig. 6 shows the query time of the algorithm under different number of query constraints. From the figure, we can observe that the time is nearly linearly proportional to the number of constraints. For a given query which has fixed 50 constraints, we assigned to all the constraints with the same transitive property and the same inference ability (length of transitive path in OWL). In 10 times experiment, we changed the path length from 1 to 10 . Fig. 7 shows the querying time is linear proportional to the length of transitive path.

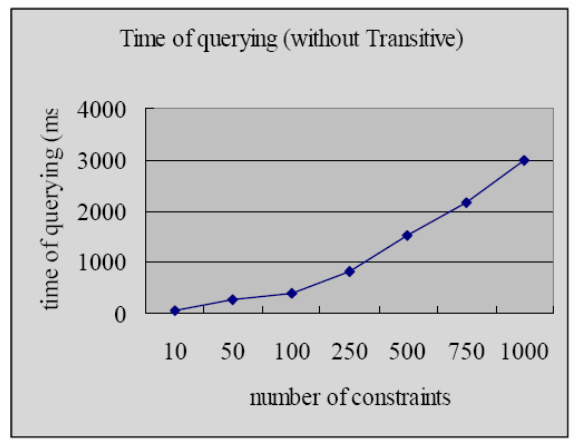

Fig. 6. The query time with different constraints

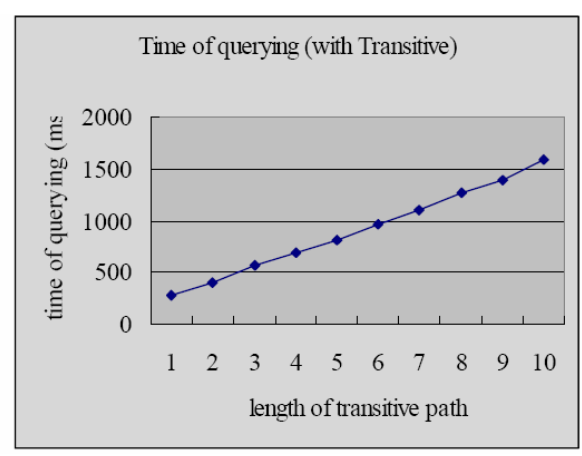

Fig. 7. The query time with different inference ability

\section{Related Work}

Several tools for persistently storing ontologies have been proposed. Protege project [3] is one of precursors for modeling and managing ontologies. The file-based storage results in that whenever an ontology is visited, the ontology has to be imported into memory. In case of the ontology size is much larger than memory, or ontology is visited frequently, this approach introduces significant overhead. Instead of storing ontologies in files, Jena 2 provides persistent ontologies storage in RDB. In this approach, each RDF statement is stored as a single row in a three column statement (subject-predicate-object) table [2]. A complete ontology class is defined by multiple RDF statements. As a result, to achieve the complete definition of an ontology class requires to combine many statements. This is also time-consuming when facing frequent query. To solve these problems, we proposed a new relational table layout for storing ontologies persistently. A number of recent research efforts have considered access control models for XML data. One of the representative efforts is the access control language, such as XML Access Control Language (XACL) [4], eXtensible Access Control Markup Language (XACML) [5] and Author-X [6] [7]. The access control language shares the feature that the language enforces an object-subjectaction-condition oriented policy on queries. [8] introduced an approach to define security views for XML document for different users. [9] adopts the encryption 
technology in securing XML document. All above approach is not suitable for the access control on OWL. In XML document, a concept is defined within an element. In case of OWL, a concept can be defined anywhere in a document. So, only securing individual elements is not enough. On the other hand, OWL document holds the inference ability. Neither of XML-targeted access control considers this issue. This paper proposes a novel approach to overcome these problems.

\section{Conclusion}

This paper contributes an approach to access control on OWL ontologies. Users can get access to an OWL document, through a specified view. Thus, the sensitive information is protected. In addition, the view specifies not only the element accessibility but also the inference ability. We also proposed the algorithm to evaluate queries against the view. The underlying persistent storage provides an efficient data retrieval. The further study will be focused on improving the view definition and refining the evaluation algorithm. The system implementation is also under consideration.

\section{References}

[1] OWL Web Ontology Language, W3C Recommendation, February 10, http:// www.w3.org/ TR/owl-features/ (2004)

[2] Wilkinson K., Sayers, C., Kuno, H., and Reynolds, D.: Efficient RDF storage and retrieval in Jena2. In Proceedings of VLDB Workshop on Semantic Web and Databases (2003) $131-150$

[3] Noy, N.F., Fergerson, R.W., and Musen, M.A.: The Knowledge Model of Protégé-2000: Combining Interoperability and Flexibility. In proceedings of 12th International Conference, EKAW 2000, Juan-les-Pins, France, October 2-6 (2000)

[4] Hada, S. and Kudo, M.: XML access control language: Provisional authorization for XML documents, http://www.trl.ibm.com/projects/xml/xacl/xacl-spec.html.

[5] OASIS, eXtensible Access Control Markup Language (XACML), http://www.oasisopen.org/committees/xacml.

[6] Bertino, E., Castano, S., and Ferrari, E.: On Specifying Security Policies for Web Documents with an XML based Language, In Proceedings of ACM SACMAT2001 (2001)

[7] Bertino, E., Castano, S., Ferrari, E.: Securing XML documents with Author-X, IEEE Internet Computing, Vol. 5, No. 3, May/June (2001) 21-31

[8] Fan, W., Chan, C.-Y., and Garofalakis, M.: Secure XML Querying with Security Views, SIGMOD 2004, Paris, France, June 13-18 (2004)

[9] Geuer-Pollmann, C.: XML Pool Encryption, ACM Workshop on XML Security, Fairfax, VA, USA, November 22 (2002) 